\title{
Kinder und Jugendliche im Umgang mit digitalen Medien und mediatisierter sexualisierter Gewalt präventiv stärken
}

Die Falldiskussionen der Kap. 4 bis 10 widmen sich Maßnahmen der Intervention, sprich Maßnahmen, um Gefahren abzuwenden und Verletzungen sowie Folgeschäden zu reduzieren. Nach Braun (2016) stellt die Befähigung von Erwachsenen zur Intervention eine Form der Prävention dar. Prävention sei in Anlehnung an Caplan (1964) als das Vorbeugen (primär) und frühzeitige Erkennen bzw. Aufdecken (sekundär) sowie das Minimieren von Folgeschäden (tertiär) sexualisierter Gewalt in Kindheit und Jugend verstanden. Angesichts der Schnittstelle von Intervention und Prävention muss Letztere Prinzipien der (Krisen-)Intervention berücksichtigen. Das setzt sowohl Wissen über Gewaltdynamiken als auch darüber voraus, wie es Betroffenen und ihrem sozialen Umfeld geht. Mit diesem Schlusskapitel beschäftigen wir uns deswegen mit Maximen gelingender Prävention mediatisierter sexualisierter Gewalt innerhalb der pädagogischen Praxis. ${ }^{1}$ Im Diskurs zu sexualisierter Gewalt bezeichnet Prävention sowohl pädagogisches Handeln mit den Zielgruppen als auch schützende Einrichtungsstrukturen. Unser Schwerpunkt liegt auf Überlegungen zum pädagogischen Handeln. Institutionelle Strukturen werden nur punktuell behandelt.

Im Sinne der Autonomie und Selbstbestimmung unserer Adressat*innen sprechen wir uns dafür aus, Maßnahmen zur Prävention an den Lebenslagen und -wirklichkeiten in Kindheit und Jugend auszurichten. Eine selbstbestimmte Nutzung digitaler Medien gehört selbstverständlich dazu (s. Kap. 1 und 2). Aktuell finden sich vereinzelte Broschüren, die Kinder und Jugendliche nicht vornehmlich auf die Gefahren der „digitalen Welt“ hinweisen. Meist beschränken sie sich auf Risiken sexualisierter Kommunikation (Sexting), das Hochladen und Teilen von

\footnotetext{
${ }^{1}$ Vornehmlich adressieren wir die pädagogische Praxis. Doch insbesondere die Ausführungen zu Haltung fordern private wie professionelle Bezugspersonen zur Lektüre und Reflexion auf.
} 
(sexualisierten) Fotos, Cybermobbing und Groomingstrategien und adressieren Kinder und Jugendliche mit Empfehlungen zu gefahren- und risikominimierenden Verhaltensweisen (z. B. keine sexualisierten Fotos von sich selbst in digitalen Medien hochzuladen). Das erachten wir insofern als problematisch, als derlei Risikodiskurse

- oftmals nicht am tatsächlichen Medienhandeln junger Menschen orientiert sind,

- die Verantwortung für den Schutz an die jungen Menschen abgeben,

- Scham- und Schuldgefühle verstärken, indem unterschwellig suggeriert wird, sich nicht ausreichend geschützt und damit inadäquat verhalten zu haben,

- Aufdeckungsprozesse erschweren,

- die Empathie des sozialen Umfeldes mit Gewaltbetroffenen unterwandern, weil ihnen eine Mitverantwortung zugeschrieben wird.

Wir sprechen uns daher für eine kritisch-emanzipatorische Haltung Erwachsener gegenüber dem Medienhandeln von Kindern und Jugendlichen aus. Eine solche Haltung ist grundsätzlich medienpositiv und insoweit an den Bedürfnissen von Kindern und Jugendlichen orientiert, als sie einen Reflexionsrahmen für wahrgenommene Verunsicherungen, Sorgen und Risiken schafft. Sie befähigt Kinder und Jugendliche zu einem selbstbestimmten und grenzachtenden Medienhandeln.

\subsection{Zu Selbstbestimmung in mediatisierten Beziehungen befähigen}

Um Kinder und Jugendliche zu einem selbstbestimmten und grenzachtenden Medienhandeln zu befähigen, bedarf es eines entsprechenden Orientierungsrahmens: Was bedeutet selbstbestimmt? Wie kann ich digitale Medien selbstbestimmt nutzen? Wie kann ich meine Grenzen in digitalen Medien wahren? In internationalen Befragungen gestanden Kinder und Jugendliche beispielsweise ein, dass sie zwar durchaus wissen, wie sie ihr Medienhandeln vor Eltern, Lehrer*innen und anderen Erwachsenen geheim halten können, doch dass sie nur bedingt wissen, wie sie das Internet geschützt nutzen können (Martin et al., 2018). Umgekehrt begrenzt sich Medienerziehung und -pädagogik empirischen Untersuchungen zufolge oftmals darauf, kindliche und jugendliche Mediennutzung über Regeln, Verhaltenskodizes und Verbote zu steuern (Sander et al., 2020; Spötzl, 2019; Süss et al., 2013). Überwachungs-Apps werden zunehmend eingesetzt, um 
eine Einhaltung der Regeln zu kontrollieren und das Nichteinhalten zu sanktionieren. Kritische Stimmen der Pädagogik und Erziehungswissenschaften sehen hierin die Gefahr, dass Fürsorge in Grenzüberschreitung (z. B. Verletzung der Privat- und Intimsphäre) überzugehen droht und zugleich wechselseitiges Vertrauen und Zutrauen in junge Menschen schwächt (Wampfler, 2019). Insofern kann eine auf Verbote und Kontrolle ausgerichtete Pädagogik keine Orientierung bieten, denn das wäre so, als würde man jemanden einen Weg beschreiben, indem man ihm nur erklärt, wo er nicht hingehen darf. Zumal es auch mit „totalitären Mitteln“ keine „Garantie zur Verhinderung [Anm.: mediatisierter sexualisierter Gewalt]" geben kann (Koch, 2020, S. 11). Kinder und Jugendliche lernen ganz im Gegenteil sich unterzuordnen, statt digitale Medien eigenverantwortlich und selbstregulativ zu nutzen. Zudem wird das Umgehen elterlicher und institutioneller Kontroll- und Überwachungsmechanismen sowie die geheime Nutzung verbotener Anwendungen begünstigt. Wenn Betroffene ihr Gewalterleben auf „Regelbrüche“ zurückführen, scheuen sie sich umso mehr, sich Dritten anzuvertrauen (Kärgel \& Vobbe, 2019).

Eine Überwachung ist auch in anderer Hinsicht nicht zweckdienlich. Aus entwicklungspsychologischer und pädagogischer Betrachtungsweise sind Experimentier- und Risikoverhalten Teil des Aufwachsens. Soziale Netzwerke fungieren als soziales Kapital (Varanasi et al., 2018). Sie spiegeln Beliebtheit und Attraktivität. Die Angst vor sozialem Ausschluss begünstigt letztlich Anpassung. Junge Menschen orientieren sich am Medienhandeln ihrer Peergruppe (Staats et al., 2019). Der internationalen Forschung zufolge wächst zugleich der Druck, den Kommunikations- und Nutzungspraktiken anderer, einschließlich deren ästhetischer Ideale, zu folgen (z. B. ständige Erreichbarkeit, sexualisierte Selbstinszenierung, Online-Dating, Online-Mating) (Yau \& Reich, 2019; Geber et al., 2021; Riesmeyer et al., 2021). „Wer nicht - schnell - mitmacht, ist bereits verdächtig und gerät in sozialen Netzwerken in Gefahr: Die Zugehörigkeit zu einer normativ erlebten (Subgruppen-) Normalität wird Ziel in einer , digitalisierten' und globalisierten Welt. Das Selbstwerterleben ist eng an die Rückmeldungen der Gemeinschaft gebunden, die anerkennt oder ausschließt. [...] Moralisches Urteil richtet sich weniger danach, was richtig oder falsch ist, sondern was zur Anerkennung der Gruppe und was zur Exklusion führt." (Staats et al., 2019, S. 13 f.).

Da mediatisierte sexualisierte Gewalt zumeist in eine wie auch immer geartete soziale Beziehung eingebettet ist (s. Kap. 2 und 4), erachten wir soziale Beziehungen, die über digitale Medien geknüpft und/oder gepflegt werden, als eine 
tragende Säule zur Prävention. Junge Menschen verspüren in sozialen Beziehungen Ambivalenzen. Ihre Verunsicherungen beziehen sich dabei meist auf Fragen danach, wo ihre eigenen Grenzen liegen und wann sie wem vertrauen können:

- Welche Konsequenzen wird es für mich haben, wenn meine Freund*innen sexualisierte Fotos und Videos in sozialen Netzwerken einstellen und ich nicht? Werde ich ausgegrenzt? Bin ich dann unbeliebt(er)? Finde ich dann keine*n Freund*in?

- Andere schicken Fotos und Videos hin und her. Stimmt etwas nicht mit mir, wenn ich mich unwohl dabei fühle/das nicht so gut finde?

- Wie kann ich jemandem/muss ich anderen meine Liebe beweisen?

- Wie kann ich herausfinden, welche Absichten die andere Person hat? Wie kann ich herausfinden, ob die andere Person es ernst mit mir meint?

- Erst hat es mir Spaß gemacht, Fotos und Videos auszutauschen, ein bisschen zu flirten und zu sexten. Aber ich merke, dass ich das plötzlich nicht mehr will/dass ich manches komisch/eklig finde. Darf ich jetzt (noch) aussteigen?

- Was mache ich, wenn ich nicht so genau weiß, ob mir etwas gefällt?

- Wie kann ich ,Nein' sagen, ohne einen Kontaktabbruch fürchten zu müssen?

- Wie kann ich ,Nein“ sagen, ohne befürchten zu müssen, dass ich ausgegrenzt werde?

Maßnahmen zur Prävention sind als Reflexionsraum zu gestalten, der es jungen Menschen alters- und entwickelungsgerecht, differenz- und kultursensibel ermöglicht,

- eigene Grenzen (auch) im Zusammenhang digitaler Medien kennenzulernen,

- (mittels leibesorientierter Methoden) ein Bewusstsein für und einen Zugang zu somatischen Gefühlen (Markern) zu erlangen,

- Exit-Strategien und Grenzziehung zu erproben,

- Hinweise auf vertrauensvolle (Fern-)Beziehungen gegenüber Verhältnissen, die von Anforderungen, Be- und Abwertungen geprägt sind, auch dann zu differenzieren, wenn Kommunikation vorwiegend medial geführt wird,

- eigene Wünsche und Bedürfnisse kennenzulernen,

- eigene Vorstellungen von Freundschaft, Liebe, (Fern-)Beziehung und Intimität zu entwickeln,

- sich in Beziehung zu anderen, vor allem auch erwachsenen Ansprechpersonen, zu erleben, die Kinder und Jugendliche insoweit als Unterstützung wahrnehmen, als diese sie nicht abwerten, solidarisch sind, nach gemeinsamen Lösungen mit ihnen suchen, verlässlich und ein Schutzfaktor sind. 


\subsection{Auf das Unrecht von Verletzungen hinweisen}

Um junge Menschen zu Selbstbestimmung und Grenzwahrung zu befähigen, müssen Kinder und Jugendliche über mediatisierte sexualisierte Gewalt einschließlich Täter*innenstrategien aufgeklärt werden. Dazu ist die Unrechtmäßigkeit von Verletzungshandlungen zu fokussieren. Die folgend skizzierten Formulierungsbeispiele verstehen sich als Veranschaulichung eines angemessenen Grundtenors. Sie dienen als Anregung und Orientierung und nicht zur direkten Verwendung in Präventionsmaterialien. Sie sind weder alters-, zielgruppengerecht noch kulturund differenzsensibel ausformuliert.

- Es ist Unrecht, wenn dir jemand androht, dich nicht mehr zu mögen, den Kontakt zu dir abzubrechen, Foto-, Video- oder Audioaufnahmen von dir über digitale Medien zu veröffentlichen oder zu verbreiten, falls du das nicht möchtest.

- Es ist Unrecht, wenn jemand böse auf dich ist, weil du keine Fotos oder Videos von dir machen, einstellen oder teilen möchtest.

- Es ist Unrecht, wenn dich jemand darum bittet oder dazu auffordert, dass du dich in bestimmter Weise fotografierst oder dir vorgibt, wie du dich filmen sollst.

- Es ist Unrecht, wenn dich jemand anmacht, obwohl du dich dabei nicht wohlfühlst.

- Du hast das Recht, ein Gespräch jederzeit zu beenden. Du bist niemandem etwas schuldig.

- Niemand hat das Recht, ohne dein Einverständnis Foto-, Video- oder Audioaufnahmen von dir über digitale Medien zu veröffentlichen oder zu verbreiten.

- Es ist Unrecht, wenn dir jemand sagt oder das Gefühl gibt, du seist anders oder komisch, weil du etwas nicht möchtest oder zu tun bereit bist.

- Du entscheidest, ob und welche Fotos und Videos du von dir machen möchtest.

- Du entscheidest, wem du Fotos und Videos von dir zeigen möchtest.

- Es kann jedem einmal passieren, dass er*sie schlechte Erfahrungen in und/oder mit digitalen Medien macht. Es kann jedem einmal passieren, dass er*sie dumm angemacht wird oder dass peinliche Bilder die Runde machen. Daran bist du nicht selbst schuld. Es gibt daher keinen Grund, sich zu schämen. Du hast ein Recht auf Hilfe. 


\subsection{Medienhandeln und mediale Grenzüberschreitungen reflektieren}

In der Präventionsarbeit stellen Übungen und Spiele unseres Erachtens ein geeignetes Instrumentarium dar, um Kindern und Jugendlichen Gesprächs- und Reflexionsräume zu wahrgenommenen Verunsicherungen und belastenden Sorgen mit Blick auf ihr Medienhandeln im Allgemeinen und ihre medial geführten Beziehungen im Speziellen zu eröffnen. Im Folgenden möchten wir durch das Skizzieren eigener Ideen zu Übungen und Spielen Impulse zur methodischdidaktischen Gestaltung von Präventionsarbeit setzen.

Fotos bewerten mal anders. Ziel der Übung ist es, das Posten/Teilen von Fotos und Videos in einen Zusammenhang zum Kommentieren veröffentlichter Beiträge zu bringen. Dabei soll es weniger um die „Angemessenheit“ von Fotos und Videos gehen (Stichwort Risikodiskurs), als vielmehr darüber zu reflektieren, welche Bedürfnisse hinter dem Posten/Teilen stehen und inwieweit öffentliche Kommentierungen (un)passend sind. Die Übung empfiehlt sich vornehmlich für Jugendliche, kann jedoch für Kinder ab fortgeschrittenem Grundschulalter altersgerecht angepasst werden.

Mit der Gruppe werden nacheinander (zuvor ausgewählte) Fotos prominenter Selbstdarstellungen in sozialen Netzwerken gezogen (Alternative: Die Teilnehmenden recherchieren selbst im Internet, das setzt jedoch Regeln für die Recherche voraus). Die Teilnehmenden werden zunächst angeregt, die Perspektive der abgebildeten Person zu übernehmen. Handlungsleitend sind folgende Fragen:

- Wie möchte die abgebildete Person gesehen werden? Woran macht ihr das fest?

- Was glaubt ihr, weshalb die abgebildete Person das Foto eingestellt/geteilt hat?

- Was glaubt ihr, welche Kommentare sich die abgebildete Person wünscht?

- Was glaubt ihr, welche Kommentare sich die Person nicht wünscht?

Die Kommentare werden (schriftlich) gesammelt. Die Fotos werden weggelegt. Auf ihnen lastet nicht der Schwerpunkt der Diskussion. Die Gruppe wird hierüber informiert. Die Kommentare sind nun losgelöster Gegenstand der Auseinandersetzung. Es erfolgt also ein Perspektivwechsel, der die Praxis des Kommentierens in den Blick nimmt: 
- Wie findet ihr den Kommentar?

- Wem kann man einen solchen Kommentar schreiben? Wann wäre er eher unpassend? (Gemeint ist hier sowohl die Qualität der Beziehung als auch der Kontext, z. B. öffentlich, privat).

- Was könnten andere denken, wenn sie so einen Kommentar lesen?

- Wie geht es einer Person, die so einen Kommentar erhält?

Die Teilnehmenden setzen sich ohne pädagogischen Gefälligkeitsdruck mit den Unterschieden zwischen Grenzachtung und Grenzüberschreitung auseinander. Im Idealfall findet sowohl eine Sensibilisierung dafür statt, dass unterschiedliche Faktoren (z. B. der Bedeutungsgehalt von Äußerungen, subjektives Erleben der Adressat*in und Kontextfaktoren) darüber entscheiden, was als angemessen bzw. grenzüberschreitend erlebt wird. Die Wahrnehmungen dürfen sich durchaus unterscheiden. Die Teilnehmenden dürfen sich nicht dazu gedrängt fühlen, über ihre persönlichen Praxen und Erfahrungen zu sprechen. Die Übung erfüllt bewusst den Zweck einer Externalisierung. Die Nähe-Distanz-Regulation überlässt sie den Teilnehmenden selbst. Auch in der Diskussion zum (öffentlichen) Kommentieren muss hervorgehoben werden, dass die Kommentare nicht den Bewertungen derjenigen Übungsteilnehmer*innen gleichgesetzt werden, die sie verfasst haben. Die Kommentierungen sind schließlich das Ergebnis von Hypothesenbildung. Freilich kann während der Diskussion eine Selbstidentifikation mit Äußerungen nicht ausgeschlossen werden. Dies erachten wir aber nur dann als problematisch, wenn dadurch die Grenzen anderer Teilnehmender verletzt werden, etwa durch Abwertungen, Beleidigungen oder Bloßstellung. Es ist wichtig, dass Pädagog*innen hierauf reagieren. Andernfalls kehrt sich die Methode in ihr Gegenteil um. Sie legt dann die Unfähigkeit der Übungsleitung, den Schutz der Teilnehmer*innen herzustellen, offen. Wir orientieren uns in dem Zusammenhang an der Haltung des PETZE-Instituts für Gewaltprävention gGmbH (2015): „Voll daneben! Oft kommen Humor, Natürlichkeit und Charme besser an als dümmliche Bagger- und Anmachsprüche. [...] Sexistische Sprache beinhaltet z. B.: ätzende Sprüche über Körper und Kleidung, anzügliche Bemerkungen und eklige Witze, blöde Sprüche über sexuelle Orientierung, abwertende Bezeichnungen oder Beschimpfungen wie Nutte, Bitch, Fotze [...].“

Im Falle von Interventionen sollten diese beim grenzverletzenden Verhalten selbst ansetzen und nicht die übergriffigen Teilnehmenden als Personen moralisch abwerten. Das grenzverletzende Verhalten ist zu benennen, indem die pädagogische Fachkraft eine Resonanz gibt, weshalb sie eine Äußerung oder ein Verhalten als verletzend erlebt oder weshalb diese nicht als Spaß einzuordnen sind, sondern eine Abwertung darstellen. Auf keinen Fall sollte reziproke Pädagogik betrieben 
werden (,Wie würde es dir gehen, wenn jemand das zu dir sagt?“). Diese erlaubt Gefälligkeitsschemata, Bagatellisierungen und einen Wechsel ins Lächerliche („Ich würde mich freuen, wenn ...“). Im Falle von Interventionen entscheiden die pädagogische Erfahrung und Haltung. Diskussionsförderliche Provokationen sollten nicht zu früh oder vehement unterbunden werden. Demgegenüber dürfen subtile Formen der Unterwerfung, die als „Spaß“ dargestellt werden, nicht unbeachtet bleiben. Für Fans einer bestimmten Sportlerin oder eines bestimmten Sängers kann es verletzend sein, wenn andere Teilnehmende behaupten, der*die Prominente bezwecke mit seinem*ihrem Social-Media-Auftritt, sexuell belästigt zu werden.

Warum ist es manchmal schwer, ...? Diese interaktive Reflexion mit Kindern, Jugendlichen oder wahlweise Pädagog*innen und Eltern setzt bei (mit Mediennutzung verbundenen) Ambivalenzen junger Menschen an. Sie verkürzt Prävention nicht auf die Empfehlung, keine Fotos von sich zu verschicken. Sie legt den Fokus auf die Schwierigkeit, die eigenen Grenzen (er)kennen zu lernen und herauszufinden, ob wir dasselbe wollen wie andere, wie wir eigentlich entscheiden, dass wir jemandem vertrauen wollen, wie wir uns fühlen, wenn wir unsicher sind und unter welchen Umständen es uns schwerfällt, unsere eigenen Gefühle, Wünsche und Bedürfnisse gegen die Erwartungen anderer durchzusetzen. Dabei wird das eigene Handeln ebenso in den Blick genommen wie das Handeln Dritter. Methodisch kann unterschiedlich vorgegangen werden. Zentral ist eine Sammlung von Fragen, die bei den Problemlagen junger Menschen ansetzt. Der Fragenkatalog sollte am Entwicklungsstand der Zielgruppe orientiert sein und lediglich Akzente setzen. Es können aus der Gruppe anonym weitere Fragen gesammelt werden. Die Sammlung kann per Zettel in einem Briefkasten oder mittels eines Umfragetools erfolgen. Beispielhafte Fragen können lauten:

- Woran mache ich für mich fest, dass jemand ein*e Freund*in ist, dem*der ich vertrauen kann?

- Wie sieht für mich die perfekte Liebesbeziehung/Fernbeziehung aus?

- Woran merke ich/Wo im Körper merke ich, dass ich etwas nicht will oder unsicher bin, ob mir etwas gefällt?

- Warum ist es manchmal total schwer, zu irgendetwas Nein zu sagen, z. B. Fotos von sich zu posten, Nacktfotos an jemanden zu senden, bei Sex mitzumachen oder sich in Chats über Sachen zu unterhalten, obwohl man nicht richtig Lust dazu hat?

- Wie verhält sich der*die perfekte Freund*in, wenn man ihm*ihr sagt, dass man nicht richtig Lust auf irgendetwas hat? 
- Wie geht es Kindern und Jugendlichen, die dem*der Falschen vertraut haben oder die etwas mitgemacht haben, obwohl sie sich unsicher waren?

- Wer ist denn verantwortlich dafür, wenn jemand dem*der Falschen vertraut?

- Wie sollten sich Freund*innen oder Erwachsene verhalten, wenn man ihnen davon erzählt?

Erwachsenen bzw. Pädagog*innen empfehlen wir zu diesen Fragen wie folgt Haltung zu beziehen: „Manchmal geben Mädchen und Jungen Dateien im Vertrauen an Freundinnen, Freunde oder an Personen, in die sie verliebt sind oder denen sie imponieren wollen. [...] Vermeiden Sie pauschale Urteile. Machen Sie [...] keine Vorwürfe, wenn etwas schiefgelaufen ist. [...] Der Austausch von SextingFotos in Beziehungen gehört bei einigen zu einer selbstbestimmten Partnerschaft. Unterstützen Sie [...], eine unangenehme Situation zu beenden.“ (PETZE-Institut für Gewaltprävention gGmbH et al., 2015).

Gerade der Austausch über potenzielle Verletzungserfahrungen sollte externalisiert werden, indem die Teilnehmenden nicht zu Selbstkundgaben angeregt werden. Dazu wird der Perspektivwechsel angeregt, „Wie geht es jemandem, der*die...?“ oder „Was würdest du einem Freund raten, wenn...?“. Trotzdem muss damit gerechnet werden, dass Teilnehmende erlebte Grenzverletzungen realisieren und sich pädagogischen Fachkräften anvertrauen. Sie sollten daher auf Aufdeckungsprozesse eingestellt sein.

Opfermythen im Check. Aus dem Forschungszusammenhang des HUMANProjekts geht hervor, dass Opfermythen, Victim-Blaming aber auch Bagatellisierungen die Perspektiven junger Menschen auf Gewalt mitbestimmen können. Unseres Erachtens spiegelt sich der pädagogische Bias, dass Kinder und Jugendliche vor den Risiken digitaler Medien achtgeben sollen, in jugendlichen Interpretationen von Glaubenssätzen wider, dass Betroffene ,dumm sind, wenn sie den Falschen vertrauen“, „,ich nicht wundern brauchen, dass sie belästigt werden, wenn sie sexy Bilder von sich posten“ oder sogar "schlampenhaftes Verhalten“ Übergriffe bedingt. Paradoxerweise schilderten uns Kinder und Jugendliche, dass in ihrer Peergroup Normen und Vorstellungen von Beziehungen gelten, durch die sie sich bisweilen gezwungen sehen, Nacktfotos von sich zu verschicken. Die Unfreiwilligkeit verstärkt Ambivalenzen, die durch Opfermythen anschlieBend bestätigt werden und eine Aufdeckung hemmen. Eine Beschäftigung mit Opfermythen kann demgegenüber ein Mittel sein, um Aufdeckung zu erleichtern und die Solidarität mit Gewaltbetroffenen zu stärken. Der Ansatz setzt abstrakte Kritik- und Reflexionsfähigkeit voraus. Er eignet sich daher eher für Jugendliche oder muss an die Reflexionsfähigkeit von Kindern angepasst werden. 
Die Diskussion zu verbreiteten Opfermythen und Bagatellisierungsstrategien wird angestoßen, indem vor allem deren Folgen reflektiert werden. Anregungen für die Reflexion von unter Jugendlichen verbreitete Mythen - wenn auch nicht auf sexualisierte Gewalt bezogen - gibt die Broschüre „FAQ You“ von Jugend gegen Aids e. V. (Wolf et al., 2019). Verbreitete Opfermythen lauten beispielsweise:

- „Typische Opfer ziehen Gewalt an.“

- „Selbst schuld, wenn man den Falschen vertraut.“

- „Wer Nacktfotos von sich verschickt, ist eine Schlampe und braucht sich nicht wundern, wenn die Bilder die Runde machen.“

- „Boys will be boys. Ist doch klar, dass man seine Trophäen rum zeigt.“

In der anschließenden Reflexion soll es weniger um den Wahrheitsgehalt der Opfermythen, sondern vielmehr um die Frage danach gehen, welchen Zweck solcherlei Annahmen erfüllen und welche Konsequenzen sie für Betroffene nach sich ziehen:

- Was sind Situationen, in denen so etwas gesagt wird?

- Welche Behauptungen verstecken sich noch in der Aussage? Wie kann man diese Behauptungen anders formulieren?

- Über wen wird hier geredet und über wen nicht?

- Wem nützt die Behauptung? Wem nützt sie nicht?

- Was nützt sie denjenigen, über die geredet wird?

- Wie geht es denen, über die geredet wird?

- Welche Message würden diejenigen, über die geredet wird, stattdessen brauchen?

Not yours to share ${ }^{2}$ ist eine Kampagne der schottischen Regierung, die entlang von vier Fallbeispielen anschaulich für das Unrecht des nicht konsensuellen Weiterleitens sexualisierter Foto- und Videoaufnahmen sensibilisiert und dabei über die Verantwortlichkeit der Gewaltausübenden sowie Hilfs- und Unterstützungsangebote aufklärt. Die Kampagne folgt einem täter*innenpräventiven Ansatz, der insofern bemerkenswert ist, als neben der Perspektive Betroffener auch auf Motive Gewaltausübender eingegangen wird. Zum Beispiel kommentiert der fiktive Täter in einem Gruppenchat das nicht konsensuell verschickte Nacktbild einer

\footnotetext{
${ }^{2}$ Die Kampagne ist unter folgendem Link einsehbar: https://notyourstoshare.scot/\#Cas eFiles.
} 
Frau mit: „Lads, how lucky am I?“ (Dt.: Jungs, was habe ich für ein Glück?). Die Kampagne erlaubt es also, sich mit unterschiedlichen Gründen für gewalttätiges Verhalten zu beschäftigten. Trotzdem bleibt in den Fallbeispielen kein Zweifel an der Unrechtmäßigkeit der Übergriffe und der Verletzung Betroffener. Wir halten es für denkbar, mit Jugendlichen entlang vergleichbarer Beispiele zu arbeiten. Ein direkter Übertrag könnte durch die Unterschiede der nationalen Rechtslage und durch Sprachhemmnisse entstehen.

\subsection{Achtsame Mediennutzung bei Gewaltbetroffenheit}

Von mediatisierter sexualisierter Gewalt betroffene Kinder und Jugendliche erleben die Nutzung digitaler Medien mitunter als dilemmatisch. Sie wähnen sich in einem Spannungsfeld zwischen Entlastung (z. B. soziale Teilhabe, Zugehörigkeit, Unterhaltung, Ablenkung, Sicherheit, Kontrolle) und Belastung (z. B. Täter*innen-Opfer-Begegnung, Trigger, Reaktivierung, Reviktimisierung, Retraumatisierung) (s. Kap. 6, 8 und 9). Umso wichtiger sind Bestärkung und Befähigung. Betroffenheit darf nicht zum Zwang einer Medienabstinenz führen. Es sind Gewaltbetroffene selbst, die darüber mitentscheiden, ob und in welcher Weise ihnen digitale Medien guttun. Aufgabe der (Sekundär- und Tertiär-) Prävention ist es, betroffene Kinder und Jugendliche entsprechend zu befähigen. Experimentelle Suchbewegungen sind hierbei ebenso in Ordnung wie Rückschläge. Vorbeugendes Handeln setzt daher eine Beschäftigung mit jenen Dilemmata voraus, die betroffene Kinder und Jugendliche hinsichtlich der Nutzung digitaler Medien häufig erleben. Wir verorten diese Beschäftigung entweder in Angeboten der Einzelfallhilfen oder Gruppenangeboten für gewaltbetroffene junge Menschen. Wichtig sind entsprechend Kompetenzen der Traumaarbeit:

- Wofür nutzt du digitale Medien?

- Wann macht es dir besonders viel Spaß, digitale Medien zu nutzen?

- Was machst du in digitalen Medien am liebsten?

- Wie fühlst du dich, wenn du in digitalen Medien unterwegs bist?

- Wann ist es denn mal nicht so schön, digitale Medien zu nutzen?

- Gibt es etwas, dass du gar nicht gerne magst oder machst in den digitalen Medien?

- Wenn du mal an die Situation zurückdenkst, in der du digitale Medien nicht so gerne genutzt hast/du dich beim Nutzen digitaler Medien nicht so gut gefühlt hast ...

- Kannst du versuchen, mir diese Situation zu beschreiben? 
- Wo im Körper hast du die unangenehmen Gefühle?

- Was war davor? Was war danach?

- Was hast du in dieser Situation gemacht?

- Wie oft hast du so etwas schon erlebt?

- Was glaubst du, würde sich für dich verändern, wenn du mal für eine Weile keine digitalen Medien nutzt? Wie kommst du darauf? Wie würde es dir gehen?

- Was wäre, wenn du nur noch die Apps und sozialen Netzwerke nutzt, die du am liebsten magst? Was würde passieren, wenn du mal eine Weile nicht in die anderen Apps und Netzwerke reinschaust?

Die Nutzung digitaler Medien ist hier eng verknüpft mit einer Psychoedukation, um erste Anzeichen für Belastungsreaktionen selbst zu erkennen und den Sog derselben zu unterbrechen.

\subsection{Medienpositive Haltung und vorbildliche Mediennutzung}

Wir sehen Erwachsene in der Co-Verantwortung, Kindern und Jugendlichen ein Vorbild im Umgang mit digitalen Medien zu sein. Provokativ stellen wir die Frage, ob wir erwarten können, dass Kinder und Jugendliche Foto- und Videoaufnahmen über digitale Medien risikobewusst, grenzachtend austauschen und potenzielle Grenzüberschreitungen Dritter frühzeitig erkennen, wenn innerhalb ihres Umfelds ganz selbstverständlich Fotos und Videos ohne das Einholen ihres Einverständnisses geteilt werden? Frantz et al. (2016) sehen in der Alltagspraxis „ganz unbefangen das eigene Familienglück medial sichtbar zu machen“ sowohl die Rechte von Kindern und Jugendlichen als auch den Schutz ihrer Privatsphäre ,untergeordnet und bisweilen [verletzt].“ (ebd., S. 2). Auch jüngere Forschungsarbeiten stellen die elterliche Vorbildfunktion infrage. Zwar geben jüngsten internationalen Studien zufolge Eltern mehrheitlich an, ihren Kindern eine ,angemessene Nutzung mobiler Geräte“ vorzuleben. Doch proklamieren $51 \%$ der befragten Teenager, dass ihre Eltern während gemeinsamer Gespräche wiederkehrend von auf dem Smartphone eingehenden Anrufen, Nachrichten und Push-Mitteilungen abgelenkt werden und teils das Gespräch dabei - das Smartphone fokussierend - fortführen (Nicolas, 2019). Auch setzen sich Jüngere im Vergleich zu Älteren häufiger mit den Privatsphäre-Einstellungen sozialer Netzwerke auseinander (Gardner \& Davis, 2014). Derlei Medienpraktiken Erwachsener vergegenwärtigen die Ähnlichkeiten zur digitalen Jugendkultur. 
In der Tat zeichnen sich in der Altersgruppe der 14- bis 59-Jährigen keine nennenswerten alters-, generations- oder kohortenspezifischen Unterschiede im Medienhandeln ab (Klein, 2017).

\begin{abstract}
„Komm schon, ich will auch sexy aussehen auf meinem Profilbild da, na hallo, was ist denn da los? Aber wir Erwachsenen nehmen doch auch keine Profilbilder von uns, wo wir bescheuert aussehen, oder? Also vielleicht jetzt nicht unbedingt sexy im Sinne von ,mhm', aber ich nehme doch auch eins, wo ich mir denke, naja, sehe ich aber gut aus.“
\end{abstract}

Traumafachberaterin, spezialisierte Fachberatung, FGI

Die weit verbreitete These, dass sich die digitale Jugendkultur von den Medienpraktiken Erwachsener unterscheide, beruht vornehmlich auf Stereotypen. Einen begünstigenden Beitrag leisten medial überzeichnete Bilder von jugendlichen Lebenswelten, die mit nostalgischen Erinnerungen an die eigene Jugend wenig gemein haben (Charteris et al., 2018; Raghunandan, 2018; Walrave et al., 2018). Medienhandeln stellt vielmehr eine Frage medialer Sozialisation und weniger eine Frage des Alters dar. Ob technische Innovationen und Trends das Medienhandeln nachhaltig prägen, ist ungeklärt (Burgfeld-Meise \& Meister, 2020). Schreiben Erwachsene jungen Menschen kollektive Verhaltensweisen pauschal zu oder grenzen sie sich dagegen betont ab, riskieren sie in puncto medialen Erfahrens und Erlebens einen Kontakt- und Beziehungsabbruch zu jungen Menschen. Infolgedessen fühlen sich Kinder und Jugendliche nicht unbegründet nur bedingt ernst genommen (s. Kap. 2), zumal die derzeit jüngste Elterngeneration bereits ähnlich medial sozialisiert wurde. Ein erhobener Zeigefinger symbolisiert in Anbetracht des Medienhandelns Erwachsener für Kinder und Jugendliche lediglich Autorität, nicht aber Authentizität.

Eine weitere tragende Säule der Prävention ist damit eine medienpositive Haltung, die wir in Erweiterung des Verständnisses von Albrecht (2017) als professionellen Kompass verstehen, der sich an der theoriegeleiteten und reflexiven Auseinandersetzung mit jenen Einstellungen und Werten ausrichtet, die (berufs-)biografisch erwachsen sind. „In einem Zeitalter, in dem es kaum mehr medienlose Räume gibt und die Mediensphäre die gesamte gesellschaftliche Wirklichkeit durchdringt" (Moser, 2019, S. 69 f.), sollte die Kompassnadel tendenziell medienbejahend ausschlagen. 


\section{Eine medienpositive Haltung}

- Eine medienpositive Haltung akzeptiert und toleriert, dass digitale Medien für die Phasen der Kindheit und Jugend entwicklungsförderlich und teilhabenotwendig sind.

- Eine medienpositive Haltung fokussiert auf die Chancen und Potenziale digitaler Medien, ohne dabei ihre Problematiken zu übersehen.

- Eine medienpositive Haltung motiviert Kinder und Jugendliche zur und bestärkt sie in der (selbstbestimmten) Nutzung digitaler Medien.

- Eine medienpositive Haltung drückt sich gegenüber Kindern und Jugendlichen in medialer Zugewandtheit und Interesse an ihrem Medienhandeln aus.

- Eine medienpositive Haltung drückt sich gegenüber Kindern und Jugendlichen darin aus, dass ihrem Medienhandeln vorurteilsfrei begegnet wird, um den Raum auch für medienkritische Auseinandersetzungen zu eröffnen.

- Eine medienpositive Haltung vermittelt Kindern und Jugendlichen, dass sie inklusive ihrer Mediennutzung und ihres Medienhandelns grundsätzlich wertgeschätzt werden.

- Eine medienpositive Haltung ist darum bemüht zu verstehen, weshalb Kinder und Jugendliche für digitale Medien begeisterungsfähig sind.

„Dazu müssen wir verstehen, was Kinder und Jugendliche beschäftigt, was sie im Rahmen ihrer Mediatisierung antreibt." (Jansen-Garz, 2020, S. 13). Das geht beispielsweise mittels dialogischer Aushandlungsformate, die authentisches Interesse an den Perspektiven und Bedürfnissen junger Menschen vermitteln:

- Was findest du spannend daran, Fotos und Videos zu posten?

- Was wünscht du dir von Menschen, die die Fotos oder Videos sehen?

- Welche Sorgen spürst du manchmal?

- Welche unangenehmen Erfahrungen hast du gesammelt?

- Wer sind deine liebsten Influencer*innen? Was fasziniert dich an ihnen? Was ist echt, was fake?

- Gibt es auch Dinge, die du mit Blick auf digitale Medien kritisch betrachtest?

- Was ist besonders spannend daran, neue Leute online kennenzulernen?

- Was gefällt dir daran, über soziale Netzwerke zu flirten und dich auf ein Date zu verabreden? 
Eine weitere Säule der Prävention ist der persönliche Umgang mit digitalen Medien, der stets selbstreflexiv und selbstkritisch auf das Wahrnehmen einer Vorbildfunktion zu hinterfragen ist.

\section{Eine vorbildliche Mediennutzung}

- Eine vorbildliche Mediennutzung fordert Erwachsene, sich in den digitalen Medien so zu bewegen, wie sie es im Gegenzug von Kindern und Jugendlichen erwarten.

- Eine vorbildliche Mediennutzung reflektiert das eigene Medienhandeln stets im Hinblick auf die Wahrung und Missachtung von Grenzen, Privatsphäre sowie Verletzung der Rechte von Kindern und Jugendlichen im Speziellen und Dritten im Allgemeinen.

- Eine vorbildliche Mediennutzung setzt somit auch voraus, Kinder und Jugendliche prinzipiell zu fragen, ob Foto-, Video- oder Audioaufnahmen von ihnen angefertigt, in digitale Medien eingestellt oder aber über digitale Medien geteilt werden dürfen.

- Eine vorbildliche Mediennutzung gewährt Kindern und Jugendlichen die Möglichkeit, ihr Einverständnis jederzeit zurückzuziehen.

Für Erziehende orientiert sich eine vorbildliche Mediennutzung in Anlehnung an Wampfler (2019) am Grundsatz von Gegenseitigkeit. Wenn es also Regeln zum Umgang mit digitalen Medien gibt, müssen diese entweder auch für Erwachsene gelten, oder es sollte - weil Jugendschutz nun mal Erwachsene nicht erfasst - ein Pendant für die Regel gefunden oder Transparenz mit Blick auf die Begründung von Regeln geschaffen werden. Wird von Kindern beispielsweise eine smartphonefreie Zeit gefordert, sollten auch Eltern smartphonefreie Zeiten einhalten. Muss das Smartphone im Unterricht ausgeschaltet bleiben, sollte das nicht minder für das Lehrpersonal gelten.

\subsection{Schlussgedanken zum Setting von Prävention}

Prävention ist so wirkungsvoll wie die Strukturen, in die sie eingebettet ist. Im deutschsprachigen Raum hat sich vor diesem Hintergrund eine Debatte etabliert, die Prävention der Herstellung institutionell schützender Strukturen gleichsetzt (Elosge et al., 2019). Wir möchten die Vorstellung institutioneller Strukturen an dieser Stelle erweitern, um den Blick nicht ausschließlich auf die Meso-Ebene 
pädagogischer Einrichtungen zu verengen. Wir verwenden Setting als Begriff für sozialökologische Faktoren, die zur Verfügung stehende räumliche und zeitliche Ressourcen, Kulturen, Normen, Leitsätze, Zeitgeist, Atmosphäre sowie Beziehungsgestaltung jenseits institutioneller Ebenen oder Zuständigkeiten umfassen (Wood et al., 2016). Wir beginnen trotzdem mit dem Beispiel pädagogischer Einrichtungen. Den Blick auf größere gesellschaftliche Zusammenhänge öffnen wir zum Schluss.

Setzen wir voraus, in einer Einrichtung gäbe es eine pädagogische Fachkraft, die sich intensiv mit mediatisierter sexualisierter Gewalt beschäftigt, Grundsätze der Krisenintervention verstanden hat und den Adressat*innen mit einer präventiven Haltung entgegentritt, so sind die Erfolge einer nachhaltigen Stärkung und eines nachhaltigen Schutzes trotzdem nicht unabhängig von dem sie umgebenden Umfeld. Zwar kann nicht ausgeschlossen werden, dass einzelne Adressat*innen von der Haltung der pädagogischen Fachkraft profitieren und sich ihr vertrauensvoll zuwenden. In einer Einrichtung mit häufigen Personalwechseln, die über kein medien- und sexualpädagogisches Konzept verfügt, Grenzverletzungen nicht entgegenwirkt und die sich um ihre Außenwahrnehmung, ihren Ruf mehr sorgt als um das Wohlergeben ihrer Adressat*innen, mag das Bemühen der Fachkraft zwar nicht gänzlich wirkungslos sein. Aller Wahrscheinlichkeit wird sie bei Problemlagen der Adressat*innen vermehrt angesprochen, nicht zuletzt aufgrund ihrer Verlässlichkeit. Dennoch wird der Nachhall ihrer präventiven Bemühungen höchstwahrscheinlich immer wieder durch gegenteilige Erfahrungen der Kinder und Jugendlichen konterkariert, zumal die betreffende Fachkraft selbst in mehrerlei Hinsicht belastet wird. Die Problemlagen der Adressat*innen liegen ausschließlich auf ihren Schultern. Sie stellt fortwährend fest, dass ihre Unterstützungsangebote nur bedingt nützen, weil sie ihre Gültigkeit verlieren, sobald die Kinder und Jugendlichen den Raum wechseln. Mit Blick auf ihr eigenes Überlastungserleben nützt es ihr nur wenig, zu wissen, dass sie nicht die Verantwortung für institutionelle Mängel tragen kann. Womöglich resigniert sie und wechselt früher oder später die Einrichtung oder sie gibt auf.

Die Prävention mediatisierter sexualisierter Gewalt bedarf einer Klammer, dem präventiven Setting. Das Setting soll gewährleisten, dass die Stärkung und der Schutz der Adressat*innen als gemeinschaftliches Ziel verstanden werden. Zwischenzeitlich existieren zahlreiche Empfehlungen, mit denen Schutzkonzepte partizipativ entwickelt werden sollen. Die Empfehlungen umfassen regelmäßige Präventionsangebote für Kinder und Jugendliche, eine Vermittlung von Kinderrechten, verbindliche Regeln des institutionellen Miteinanders, Verfahrenswege zum Umgang mit Hinweisen auf oder das Bekanntwerden von Grenzverletzungen, Zuständigkeiten sowie Verantwortlichkeiten (Elosge et al., 
2019). Interessanterweise werden digitale Medien als Kontext sexualisierter Gewalt hierbei nach wie vor eher zweitrangig behandelt. Man könnte meinen, dass genau hierin die Implementierungsleistung der jeweiligen Einrichtung läge, da sich ihre institutionellen Voraussetzungen zu sehr für Verallgemeinerungen unterscheidet. Dies halten wir jedoch für eine Schutzbehauptung, die ignoriert, welchen Stellenwert digitale Medien im Alltag junger Menschen und auch für pädagogisches Handeln selbst haben. Der Verweis auf das Recht am eigenen Bild allein, Netiquette für Klassenchats, Kodizes, dass keine privaten OnlineKontakte zwischen Professionellen und Adressat*innen stattfinden, oder wie die Datenschutzgrundverordnung (DSGVO) auszulegen sei, sind wichtige Handlungsgrundsätze. Sie werden der Komplexität der Herausforderungen aber bei Weitem nicht gerecht. Leider ist die Zahl der Projekte, die sich einer zeitgemäßen Mediatisierung pädagogischer Einrichtungen widmet, gering. Letzteres war nicht der Auftrag des HUMAN-Projekts, deswegen sei an dieser Stelle auf das BMBFgeförderte Projekt DigiPäd24/7 verwiesen. Die Technische Hochschule Köln und die Universität Hildesheim erforschen im Verbund mit stationären Jugendhilfeeinrichtungen Richtlinien und Handlungsempfehlungen zur Entwicklung von Handlungskonzepten zum Medienalltag von Kindern und Jugendlichen in Heimen und Internaten, der Veränderung der Erziehungspartnerschaften zwischen Eltern und Fachkräften sowie dem rechtlichen Handlungsrahmen der Einrichtungen im Bereich der Medienbildung. Darüber hinaus entwickelt das ebenfalls BMBF-geförderte Projekt SaferSexting der Europa Universität Flensburg und des Universitätsklinikums Hamburg-Eppendorf gendersensible Lernmodule für die hochschulische Aus- und Fortbildung von Lehrkräften an weiterführenden Schulen, um junge Menschen im Hinblick auf den Umgang mit mediatisierten sexualisierten Grenzverletzungen besser zu begleiten. Die Ergebnisse des HUMAN-Projekts bilden ferner die Grundlage des von der European Commission geförderten Projekts Beyond Digital Violence: Capacity Building for Relevant Professionals Working with Children and Young People Who Experienced Sexualised Violence Using Digital Media (ByeDV). Die SRH Hochschule Heidelberg und die Deutsche Gesellschaft für Prävention und Intervention bei Kindesmisshandlung, -vernachlässigung und sexualisierter Gewalt e. V. implementieren die Handlungsempfehlungen gemeinsam mit spezialisierten Facheinrichtungen in der Praxis. Hieraus werden Qualitätskriterien für die Implementierung abgeleitet.

Ein weiterer Aspekt der Überlegungen zu präventiven Settings betrifft den digitalen Raum selbst. In diesem Zusammenhang bleiben Potenziale ungenutzt. Zwar werden seit der Einführung der DSGVO verstärkt Bemühungen unternommen, die Daten von Nutzer*innen und deren Verarbeitung besser zu schützen. Das ist einer Prävention sexualisierter Gewalt nicht gleichzusetzen. Für Letztere 
fehlt die flächendeckende Unterstützung der Anbieter*innen, welche sich oftmals auf Altersschutzgrenzen für die Nutzung ihrer Angebote zurückziehen. Allerdings gibt es zahlreiche Angebote, die entsprechend der Altersschutzgrenze für Kinder und Jugendliche ungeeignet wären, deren Nutzer*innen sich aber zum Großteil genau aus dieser Altersgruppe speist. Ungenutzt bleiben die finanziellen und technischen Möglichkeiten, welche soziale Netzwerke, Messenger, Suchmaschinen, Videoportale und Spieleanbieter hätten, um junge Menschen algorithmusbasiert mit genau den Botschaften anzusprechen, die Betroffenen helfen, ganze Armeen von Influencer*innen für die Stärkung junger Menschen ins Feld zu führen, mittels Geolocation die nächstgelegene Beratungsstelle zu vermitteln, mittels Big Data Täter*innenstrategien auszuwerten, Online-Beratung anzubieten oder im Umfang der auf der eigenen Plattform verübten sexualisierten Übergriffe existierende Beratungslandschaften finanziell zu unterstützen. Für diese Utopie setzt sich der Stab des Unabhängigen Beauftragten für Fragen des sexuellen Kindesmissbrauchs ein. Im Rahmen dieser Bestrebungen könnte er politische Unterstützung gebrauchen. Darüber hinaus existieren Vereine wie Freiwillige Selbstkontrolle Multimedia-Dienstanbieter e. V. (FSM), eine Selbstkontrolleinrichtung, der Anbieter*innen beitreten können, um Jugendmedienschutz zu stärken und illegale, jugendgefährdende und entwicklungsbeeinträchtigende Inhalte in Onlinemedien einzudämmen. Der Verein verfügt über eine Beschwerdestelle, bietet Beratung an und verknüpft Medienpädagogik mit technischen Möglichkeiten des Jugendschutzes. Aktuell sind dort vor allem Streamingdienste Mitglied. Die Zielsetzungen des Vereins sind stark am Staatsvertrag über den Schutz der Menschenwürde und den Jugendschutz in Rundfunk und Telemedien (JMStV) ausgerichtet. Entsprechende Initiativen könnten mit einer Erweiterung um von jungen Menschen genutzte soziale Medien und einer Veränderung ihres Selbstverständnisses Beiträge zur Weiterentwicklung präventiver Settings leisten. Bis dahin bewegen sich die vorliegenden Handlungsempfehlungen im vorgegebenen Rahmen.

\section{Literatur}

Albrecht, R. (2017). Beratungskompetenz in der Sozialen Arbeit: Auf die Haltung kommt es an. Kölner Zeitschrift für Soziologie und Sozialpsychologie, 48(1), 45-64.

Braun, B. (2016). Prävention sexualisierter Gewalt - Verständnis und Haltung. Kindesmisshandlung und-vernachlässigung, 19(2), 194-199.

Burgfeld-Meise, B., \& Meister, D. M. (2020). Generationsspezifische Medienzugänge. In T. Fuchs, A. Schierbaum, \& A. Berg (Hrsg.), Jugend, Familie und Generationen im Wandel (S. 329-343). Springer VS. 
Charteris, J., Gregory, S., \& Masters, Y. (2018). ,Snapchat', youth subjectivities and sexuality: Disappearing media and the discourse of youth innocence. Gender and Education, 30(2), 205-221.

Elosge, M., Pöter, J., Humme, M., \& Wazlawik, M. (2019). Schutzkonzepte und pädagogische Praxis: Diskursanalytische Perspektiven auf die Prävention von sexualisierter Gewalt in Einrichtungen der stationären Kinder- und Jugendhilfe (SCHUPPS). Soziale Passagen, 11(1), 209-214. https://doi.org/10.1007/s12592-019-00307-3.

Frantz, A., Hajok, D., \& Lauber, A. (2016). Wenn Eltern Bilder ihrer Kinder online stellen. Kinderrechte und Elternpflichten im Kontext des Kinder- und Jugendmedienschutzes. Jugend Medien Schutz-Report, 39(6), 2-6. doi: https://doi.org/10.5771/0170-5067-201 6-6-2.

Gardner, H., \& Davis, K. (2014). The app generation: How today's youth navigate identity, intimacy, and imagination in a digital world; with a new preface. New HavenYale University Press.

Geber, S., Baumann, E., Czerwinski, F., \& Klimmt, C. (2021). The effects of social norms among peer groups on risk behavior: A multilevel approach to differentiate perceived and collective norms. Communication Research, 48(3), 319-345. https://doi.org/10.1177/009 3650218824213

Jansen-Garz, G. (2020). Digitalisierung und Jugendschutz: Interview mit Katharina Kärgel und Frederic Vobbe. THEMA JUGEND, 4, 12-14.

Kärgel, K., \& Vobbe, F. (2019). 7 Thesen zu sexualisierter Gewalt mit digitalem Medieneinsatz. Pädagogische Rundschau, 73, 391-410.

Klein, A. (2017). Der Weg ins digitale Netz - Eine Frage des Alters und der Generation? Ergebnisse einer qualitativen Studie zur Bedeutung von Alters- und Generationsstereotypen für den Umgang mit digitalen Medien. In K. Mayrberger, J. Fromme, P. Grell, \& T. Hug (Hrsg.), Jahrbuch Medienpädagogik 13 (S. 61-77). Springer VS.

Koch, S. (2020). „Mehrfachbelastung und Reviktimisierung sind häufige Folgen“. report psychologie, 45(10), 9-11.

Martin, F., Wang, C., Petty, T., Wang, W., \& Wilkins, P. (2018). Middle school students' social media use. Journal of Educational Technology \& Society, 21(1), 213-224.

Moser, H. (Hrsg.). (2019). Einführung in die Medienpädagogik. Springer VS.

Nicolas, É. M. (2019). The Impact of Social Media on Attachment Style for Generation Z. Dissertation, Alliant International University, San Francisco.

PETZE-Institut für Gewaltprävention gGmbH. (2019). Senden!? Teilen!? Mehr Sicherheit im Umgang mit Kinderfotos im Netz! https:/www.petze-institut.de/wp-content/uploads/ 2019/01/Postkarte_Kinderfotos_Web.pdf. Zugegriffen: 13. Mai 2021.

PETZE-Institut für Gewaltprävention gGmbH. (2015). Wo hört der Spaß auf? Infos für Jugendliche über sexuelle Selbstbestimmung und sexuelle Gewalt. https://www.petze-ins titut.de/wp-content/uploads/2016/02/2015_Wo_hoert_der_Spass_auf.pdf. Zugegriffen: 13. Mai 2021.

PETZE-Institut für Gewaltprävention gGmbH, Aktion Kinder- und Jugendschutz SchleswigHolstein e. V. und Ministerium für Soziales, Gesundheit, Wissenschaft und Gleichstellung Schleswig-Holstein. (2015). SEXTING: Informationen zu einem problematischen Medienverhalten. https://www.petze-institut.de/wp-content/uploads/2015/07/Sex ting_Elternflyer_1.6.pdf. Zugegriffen: 13. Mai 2021. 
Raghunandan, K. (2018). Young people in the digital age: Metrics of friendship. In S. Punch \& R. M. Vanderbeck (Hrsg.), Families, intergenerationality, and peer group relations (S. 415-434). Springer International Publishing.

Riesmeyer, C., Pohl, E., \& Ruf, L. (2021). Stressed, but connected. MedienPädagogik: Zeitschrift für Theorie und Praxis der Medienbildung, 17-41. doi: https://doi.org/10.21240/ mpaed/00/2021.02.10.X.

Sander, U., von Gross, F., \& Hugger, K.-U. (2020). Handbuch Medienpädagogik. Springer VS.

Spötzl, S. (2019). Digitale Medien und Medienerziehung in der Familie: Eine qualitative leitfadengestützte Untersuchung über die subjektive Sichtweise von Eltern mit Kindern zwischen null und 18 Monaten. Masterarbeit, Hochschule für angewandte Wissenschaften Landshut, Landshut.

Staats, H., Ackermann, J., \& Sarrar, L. (2019). Gruppenprozesse in digitalen Welten. Eine entwicklungspsychologische Perspektive. Gruppenpsychotherapie und Gruppendynamik, 55(1), 8-27. https://doi.org/10.13109/grup.2019.55.1.8

Süss, D., Lampert, C., \& Trueltzsch-Wijnen, C. (2013). Medienpädagogik: Ein Studienbuch zur Einführung (2. Aufl.). Springer VS.

Varanasi, R. A., Dicicco, E., \& Gambino, A. (2018). Facebook reactions: Impact of introducing new features of SNS on social capital. In C. Stephanidis (Hrsg.), HCI international 2018 - posters' extended abstracts (S. 444-451). Springer International Publishing.

Walrave, M., van Ouytsel, J., Ponnet, K., \& Temple, J. R. (Hrsg.). (2018). Sexting: Motives and risk in online sexual self-presentation. Springer International Publishing.

Wampfler, P. (2019). Macht im Netz: Vom Cybermobbing bis zum Überwachungsstaat: für die Sekundarstufe II. Reclam.

Wood, M. A., Bukowski, W. M., \& Lis, E. (2016). The digital self: How social media serves as a setting that shapes youth's emotional experiences. Adolescent Research Review, 1, 163-173.

Wolf, M., Nagel, D., Malessa, R., \& Jost, M. (2019). FAQ you-Ein Aufklärungsbuch. Jugend gegen AIDS Inhouse GmbH.

Yau, J. C., \& Reich, S. M. (2019). „It's Just a Lot of Work“: Adolescents' self-presentation norms and practices on facebook and instagram. Journal of Research on Adolescence: The Official Journal of the Society for Research on Adolescence, 29(1), 196-209. https:// doi.org/10.1111/jora.12376. 
Open Access Dieses Kapitel wird unter der Creative Commons Namensnennung 4.0 International Lizenz (http://creativecommons.org/licenses/by/4.0/deed.de) veröffentlicht, welche die Nutzung, Vervielfältigung, Bearbeitung, Verbreitung und Wiedergabe in jeglichem Medium und Format erlaubt, sofern Sie den/die ursprünglichen Autor(en) und die Quelle ordnungsgemäß nennen, einen Link zur Creative Commons Lizenz beifügen und angeben, ob Änderungen vorgenommen wurden.

Die in diesem Kapitel enthaltenen Bilder und sonstiges Drittmaterial unterliegen ebenfalls der genannten Creative Commons Lizenz, sofern sich aus der Abbildungslegende nichts anderes ergibt. Sofern das betreffende Material nicht unter der genannten Creative Commons Lizenz steht und die betreffende Handlung nicht nach gesetzlichen Vorschriften erlaubt ist, ist für die oben aufgeführten Weiterverwendungen des Materials die Einwilligung des jeweiligen Rechteinhabers einzuholen.

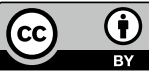

